TRANSACTIONS OF THE

AMERICAN MATHEMATICAL SOCIETY

Volume 357, Number 10, Pages 4175-4190

S 0002-9947(04)03622-0

Article electronically published on November 4, 2004

\title{
CLASSIFICATION OF REGULAR MAPS OF NEGATIVE PRIME EULER CHARACTERISTIC
}

\author{
ANTONIO BREDA D'AZEVEDO, ROMAN NEDELA, AND JOZEF ŠIRÁŇ
}

\begin{abstract}
We give a classification of all regular maps on nonorientable surfaces with a negative odd prime Euler characteristic (equivalently, on nonorientable surfaces of genus $p+2$ where $p$ is an odd prime). A consequence of our classification is that there are no regular maps on nonorientable surfaces of genus $p+2$ where $p$ is a prime such that $p \equiv 1(\bmod 12)$ and $p \neq 13$.
\end{abstract}

\section{INTRODUCTION}

A regular map is an embedding of a graph in a compact, connected surface, such that the automorphism group of the embedding acts regularly on flags (edges with a longitudinal and a transverse direction). If the surface is orientable and one requires only the orientation-preserving automorphism group of the embedding to be regular on arcs (edges with longitudinal direction), then the map is orientably regular.

The ubiquitous five platonic solids are the most well-known examples of regular maps. Nonspherical regular maps appeared in the form of stellated polyhedra in the work of Kepler 24 as early as 1619. Origins of more current interest in the study of regular maps go back to the late 19th century and were brought up by two independent streams of research. In connection with Heawood's work on map colourings that eventually led to the rise of modern topological graph theory, Heffter [18] constructed orientably regular embeddings of complete graphs of prime order. On the other hand, certain three-valent regular maps on a surface of genus three were studied by Klein [25] and Dyck [13] in connection with constructions of automorphic functions on surfaces. In the beginning of the 20th century, regular maps appeared as a form of a geometric representation of groups in Burnside's monograph [8]. The first systematic treatment of regular maps was due to Brahana [6] and was later developed on the levels of both geometry and combinatorial group

Received by the editors April 9, 2003 and, in revised form, December 11, 2003.

2000 Mathematics Subject Classification. Primary 05C10; Secondary 57M15, 57M60, 20F65, $05 \mathrm{C} 25$.

Key words and phrases. Regular maps, nonorientable surfaces, quotients of triangle groups, prime Euler characteristic.

The authors thank the Department of Mathematics of the University of Aveiro and the Research Unit "Matemática e Aplicações" for supporting this project.

The second author acknowledges support from the VEGA Grant No. 2/2060/22 and from the APVT Grant No. 51-012502.

The third author was sponsored by the U.S.-Slovak Science and Technology Joint Fund under Project Number 020/2001, and also in part by the VEGA Grant No. 1/9176/02 and the APVT Grant No. 20-023302. 
theory by Coxeter and Moser [11. Modern foundations of the theory of maps on orientable surfaces can be found in Jones and Singerman [22]. An analogous theory for maps on nonorientable surfaces was outlined by Bryant and Singerman [7].

Links between the theory of regular and orientably regular maps, group theory, hyperbolic geometry, and complex functions have been known for more than a century. In the orientable case they can be briefly summed up as follows. Automorphism groups of orientably regular maps on surfaces of genus at least two are quotients of hyperbolic triangle groups by torsion-free normal subgroups of finite index. The same quotient construction can be used to endow maps with complex structure and hyperbolic geometry from the Poincaré complex upper half-plane on which the triangle groups act. An additional boost to the study of regular maps was given lately by a part of Grothendieck's programme [17 to investigate the absolute Galois group by means of its action on maps. For more reading about these exciting connections we recommend the survey papers by Jones [21] and by Jones and Singerman 23.

In light of the above facts, classification of regular and orientably regular maps is one of the important problems whose solution would be in the interest of (and may find applications in) disciplines such as topological graph theory, group theory, hyperbolic geometry, and the theory of Riemann surfaces. The problem has been approached in three natural ways: classification by underlying graphs, by automorphism groups, and by supporting surfaces. Since our focus is on classification by surfaces we mention only the three most significant results in the first two approaches. An abstract characterization of graphs underlying regular and orientably regular maps was given by Gardiner, Nedela, Širáň and Škoviera [15]. A classification of orientably regular embeddings of complete graphs can be found in James and Jones [20]. For maps with given group, Sah [29] gave a classification of all orientably regular maps with automorphism groups isomorphic to $P S L(2, q)$.

We now briefly survey regular maps on a fixed surface. The only surfaces supporting infinitely many regular maps are a sphere, a projective plane, and a torus. The infinitude in the first two cases is due to trivial maps whose underlying graphs are cycles, dipoles, and semistars. In contrast, there is no regular map on a Klein bottle. For the remaining cases it follows from the Hurwitz bound (see Tucker 31]) that the order of the automorphism group of a regular map on a surface of negative Euler characteristic $\chi$ cannot exceed $-84 \chi$. This implies that the number of regular maps on such surfaces is automatically finite. A strengthening of the above bound to $-8 \chi+16$ for infinitely many $\chi$ follows from the work of Accola [1] or MacLachlan 26].

Regular and orientably regular maps on orientable surfaces of genus at most seven together with regular maps on nonorientable surfaces of genus at most eight have been classified by the late 1980's. This was an outcome of effort of a multitude of authors over a considerable time span (see Brahana [6], Coxeter and Moser [11, Sherk [30, Garbe [14, Bergau and Garbe [5] and the references therein). For genus two or more the main method was relation-chasing, supported by certain combinatorial arguments. With the help of the low index subgroup algorithm applied to certain finitely presented groups related to triangle groups, Conder and Dobcsányi [9] recently gave a computer-assisted classification of all regular and orientably regular maps on orientable surfaces of genus at most 15 and a classification of all regular maps on nonorientable surfaces of genus at most 30 . Thus, complete lists 
of regular and orientably regular maps were available only for a finite number of surfaces at the time of submission of this article.

A particularly interesting open question was whether there exist infinitely many nonorientable surfaces supporting no regular map at all. Conder and Everitt [10] constructed a variety of infinite families of regular maps on nonorientable surfaces, covering about 75 percent of characteristics. On the other hand, Wilson and Breda 33. proved that among all nonorientable surfaces of genus at most 52 only those of genus 2, 3, 18, 24, 27, 39 and 48 do not support a regular map.

This paper represents a breakthrough in the nonorientable regular map classification problem. We derive a complete classification of all nonorientable regular maps with negative odd prime Euler characteristic - or, equivalently, regular maps on nonorientable surfaces of genus $p+2$ where $p$ is an odd prime. As a by-product we also obtain an affirmative answer to the above question. To be able to state our main result in a condensed form, for $p \equiv-1(\bmod 4)$ we denote by $\nu(p)$ the number of pairs of coprime integers $(j, l)$ such that $j>l \geq 3$, both $j$ and $l$ are odd, and $(j-1)(l-1)=p+1$.

Theorem 1.1. Let $p$ be an odd prime, $p \neq 3,7,13$, and let $\mathcal{N}_{p+2}$ be a nonorientable surface of Euler characteristic $-p$ (and hence of genus $p+2$ ).

(1) If $p \equiv 1(\bmod 12)$, then there is no regular map on $\mathcal{N}_{p+2}$.

(2) If $p \equiv 5(\bmod 12)$, then, up to isomorphism and duality, there is exactly one regular map on $\mathcal{N}_{p+2}$.

(3) If $p \equiv-5(\bmod 12)$, then, up to isomorphism and duality, there are $\nu(p)$ regular maps on $\mathcal{N}_{p+2}$.

(4) If $p \equiv-1(\bmod 12)$, then, up to isomorphism and duality, $\mathcal{N}_{p+2}$ supports exactly $\nu(p)+1$ regular maps.

A more detailed statement of this result in group-theoretic language will appear later as Theorem 2.2 in Section 2 preceded by essentials of the theory of regular maps. In Section 3 we reduce the problem of classifying regular maps on nonorientable surfaces of Euler characteristic $-p$ ( $p$ a prime) by proving that the orders of the corresponding groups are severely limited if $p \geq 29$. Using a powerful result of Gorenstein and Walter [16] that characterizes groups with dihedral Sylow 2-subgroups, in Sections 4 and 5 we further restrict the class of candidates for automorphism groups of our regular maps. Section 6 contains a proof of Theorem 2.2 and concluding remarks.

\section{REgular MAPS AND GROUPS}

A precise definition of a map requires introducing flags or their equivalents such as oriented forms [32], or edges with longitudinal and transverse direction or blades 7. This in general requires discussing a number of degenerate cases. The objects of our interest, however, are maps that are regular and on nonorientable surfaces. For such maps, flags can be identified with (topological) triangles whose three distinguished points are a vertex, the "center" of an edge incident with the vertex, and the "center" of a face incident with both the vertex and the edge. Each face bounded by an $m$-gon is thus subdivided into $2 m$ flags.

It is well known that the automorphism group of a regular map can be generated by three involutions $x, y, z$ reflecting a fixed flag in its three sides and sending it to the three incident flags. One may choose the notation in such a way that 
the compositions $y z, z x$, and $x y$ are rotations of the map about the vertex, the center of the face, and the center of the edge associated with the fixed flag. If $k$ and $m$ are the vertex valence and the face length of $M$ we say that $M$ is of type $\{m, k\}$. In such a case the automorphism group of $M$ has a presentation of the form $\operatorname{Aut}(M)=\left\langle x, y, z \mid x^{2}=y^{2}=z^{2}=(y z)^{k}=(z x)^{m}=(x y)^{2}=\ldots=1\right\rangle$, where dots indicate a possible presence of additional independent relators. We emphasize that in all group presentations in this article, exponents will be assumed to be true orders of the corresponding elements.

Motivated by the above, we define a $(k, m, 2)$-group to be any finite group $G=$ $\langle x, y, z\rangle$ generated by an ordered triple $(x, y, z)$ of involutions and presented in the form

$$
G=\left\langle x, y, z \mid x^{2}=y^{2}=z^{2}=(y z)^{k}=(z x)^{m}=(x y)^{2}=\ldots=1\right\rangle .
$$

It follows that $(k, m, 2)$-groups are finite torsion-free quotients of the extended $(k, m, 2)$-triangle group [7. We will say that two $(k, m, 2)$-groups $G=\langle x, y, z\rangle$ and $G^{\prime}=\left\langle x^{\prime}, y^{\prime}, z^{\prime}\right\rangle$ are congruent if there is a group isomorphism from $G$ onto $G^{\prime}$ taking $x$ to $x^{\prime}, y$ to $y^{\prime}$, and $z$ to $z^{\prime}$.

Any $(k, m, 2)$-group $G=\langle x, y, z\rangle$ acts on a (unique) closed surface as the automorphism group of a regular map $M=(G ; x, y, z)$ of type $\{m, k\}$. Flags of $M$ are elements of $G$, and edges, vertices and faces of $M$ are left cosets of the dihedral subgroups $\langle x, y\rangle,\langle y, z\rangle$, and $\langle z, x\rangle$ of $G$, respectively. Mutual incidence of the map elements is given by nonempty intersection, and $G$ acts on $M$ as a map automorphism group by left multiplication. This, in principle, enables one to identify regular maps of type $\{m, k\}$ with $(k, m, 2)$-groups; a precise statement will be given in Proposition 2.1

Let $G=\langle x, y, z\rangle$ and $G^{\prime}=\left\langle x^{\prime}, y^{\prime}, z^{\prime}\right\rangle$ be a pair of $(k, m, 2)$-groups and let $M=$ $(G ; x, y, z)$ and $M^{\prime}=\left(G^{\prime} ; x^{\prime}, y^{\prime}, z^{\prime}\right)$ be the corresponding regular maps. Then, $M$ and $M^{\prime}$ are isomorphic if the groups $G$ and $G^{\prime}$ are congruent. Further, the maps $M$ and $M^{\prime}$ are dual of each other if there is a group isomorphism from $G$ onto $G^{\prime}$ which sends $x$ onto $y^{\prime}, y$ onto $x^{\prime}$, and $z$ onto $z^{\prime}$. It is easy to see that these definitions exactly correspond to the traditional concepts of map isomorphism and map duality.

Let $\mathcal{S}$ be the supporting surface of a regular map $M=(G ; x, y, z)$ and let $\chi(\mathcal{S})$ be the Euler characteristic of $\mathcal{S}$. The Euler characteristics $\chi(G)$ of the $(k, m, 2)$-group $G=\langle x, y, z\rangle$ and $\chi(M)$ of the map $M$ are defined by $\chi(G)=\chi(M)=\chi(S)$. They can be obtained from Euler's formula by substituting $|G| / 2 k,|G| / 4$, and $|G| / 2 m$ for the number of vertices, edges, and faces, respectively, giving $\chi(M)=\chi(G)=$ $(1 / k+1 / m-1 / 2)|G| / 2$.

We have the following obvious but important consequence of the above considerations.

Proposition 2.1. Regular maps on a surface of Euler characteristic $\chi \leq 2$ are, up to isomorphism and duality, in a one-to-one correspondence with congruence classes of $(k, m, 2)$-groups, $k \geq m$, of Euler characteristic $\chi$.

Given a $(k, m, 2)$-group $G=\langle x, y, z\rangle$, we will be using throughout the notation $r=y z$ and $s=z x$. The supporting surface $\mathcal{S}$ of the regular map $M=(G ; x, y, z)$ is nonorientable if and only if $G=\langle r, s\rangle$. Note that if $G$ has odd Euler characteristic (which is going to be the case in this article), then $\mathcal{S}$ is automatically nonorientable. Therefore we will also work with the reduced presentation $G=\langle r, s| r^{k}=s^{m}=$ 
$\left.(r s)^{2}=\ldots=1\right\rangle$. In general one should be aware of a loss of information when "forgetting" about the original presentation of $G$ in terms of $x, y, z$. The reason is that there may be several nonequivalent ways to choose the involutions $x, y, z$ such that $r=y z$ and $s=z x$, leading to noncongruent groups and hence nonisomorphic regular maps whose automorphism groups have the same reduced presentation. But our proofs will show that this will never be the case with the maps and groups considered here.

We will use the standard notation $Z_{n}, D_{n}, P G L(2, q)$ and $P S L(2, q)$ for a cyclic group of order $n$, dihedral group of order $2 n$, and 2-dimensional projective general and special linear group over $G F(q)$, respectively. In addition we need to introduce two special classes of groups. For any $i \geq 2$ such that $i \equiv-1(\bmod 3)$ let $G_{i}$ be the $(i+4,4,2)$-group of order $8(i+4)$ with reduced presentation

$$
G_{i}=\left\langle r, s \mid r^{i+4}=s^{4}=(r s)^{2}=s r^{3} s^{-1} r^{3}=1\right\rangle .
$$

Further, for any pair of odd integers $j \geq 3$ and $l \geq 3$ let $G_{j, l}$ be the $(2 j, 2 l, 2)$-group of order $4 j l$ with reduced presentation

$$
G_{j, l}=\left\langle r, s \mid r^{2 j}=s^{2 l}=(r s)^{2}=\left(r s^{-1}\right)^{2}=1\right\rangle .
$$

We will show later in Section 5 that the groups $G_{i}$ and $G_{j, l}$ are abstractly isomorphic to a product $Z_{i+4} \cdot D_{4}$ and to the direct product $D_{j} \times D_{l}$, respectively. Actually, both classes have appeared before. The groups $G_{i}$ are encountered, for example, in bounding the number of automorphisms of a compact Riemann surface [1, 26], while both $G_{i}$ and $G_{j, l}$ have been used for constructions of regular maps on nonorientable surfaces in [10]. The family $G_{i, j}$ also appears in constructions of groups of every strong symmetric genus [27].

The first class of groups will be of interest for us in the case when $i=p$ is an odd prime such that $p \equiv-1(\bmod 3)$. In the classification result below, the groups $G_{p}$ appear in parts (3) and (6). Among the groups $G_{j, l}$, we shall be interested in the subclass with odd and coprime parameters $j$ and $l$ such that $j>l \geq 3$ and $(j-1)(l-1)=p+1$, where $p$ is a prime satisfying $p \equiv-1(\bmod 4)$ and $p \geq 7$. We recall that the number of such pairs $(j, l)$ was denoted earlier by $\nu(p)$. The corresponding $\nu(p)$ groups $G_{j, l}$ will be referred to as groups associated with $p$; they appear in parts (4) and (6) of our classification. Observe that for $p \equiv-1(\bmod 4)$ and $p \geq 7$ we always have $\nu(p) \geq 1$ because of the pair $j=(p+3) / 2$ and $l=3$.

We are now ready to present the extended version of our main result, stated in terms of $(k, m, 2)$-groups and their reduced presentations. The reason for using group-theoretic language is twofold. First, all our proofs are purely group-theoretic. Second, the translation back to regular maps is straightforward by Proposition 2.1

Theorem 2.2. Let $p$ be an odd prime and let $n(p)$ be the number of pairwise noncongruent $(k, m, 2)$-groups $G=\langle x, y, z\rangle=\langle r, s\rangle$ of Euler characteristic $-p$ such that $k \geq m$.

(1) If $p \equiv 1(\bmod 12)$ and $p \neq 13$, then $n(p)=0$.

(2) We have $n(13)=1$, and the corresponding group is a $(7,3,2)$-group of order 1092 isomorphic to $\operatorname{PSL}(2,13)$ with reduced presentation

$$
\left\langle r, s \mid r^{7}=s^{3}=(r s)^{2}=\left(\left(s r^{-2}\right)^{4} s r^{3}\right)^{2}=1\right\rangle .
$$

(3) If $p \equiv 5(\bmod 12)$, then $n(p)=1$. The unique representative here is the $(p+4,4,2)$-group $G_{p}$. 
(4) If $p \equiv 7(\bmod 12)$ and $p \neq 7$, then $n(p)=\nu(p)$ and the corresponding groups are the $(2 j, 2 l, 2)$-groups $G_{j, l}$ associated with $p$.

(5) We have $n(7)=3$, where one of the groups is the $(10,6,2)$-group $G_{5,3}$ and the other two groups are the $(8,3,2)$-groups of order 336 isomorphic to $P G L(2,7)$ with reduced presentations

$$
\begin{gathered}
\left\langle r, s \mid r^{8}=s^{3}=(r s)^{2}=\left(s r^{-2}\right)^{4}=1\right\rangle \text { and } \\
\left\langle r, s \mid r^{8}=s^{3}=(r s)^{2}=\left(\left(s r^{-2}\right)^{2} r^{-2}\right)^{2}=1\right\rangle .
\end{gathered}
$$

(6) If $p \equiv-1(\bmod 12)$, then $n(p)=\nu(p)+1$, the groups being the $\nu(p)$ groups $G_{j, l}$ associated with $p$, together with the group $G_{p}$.

(7) Finally, we have $n(3)=4$, where one of the groups is the $(6,6,2)$-group $G_{3,3}$ and the other three groups are the $(5,4,2)$-group isomorphic to $S_{5}$, the $(5,5,2)$-group isomorphic to $A_{5}$, and the $(6,4,2)$-group isomorphic to $\left(Z_{3} \times Z_{3}\right) \rtimes D_{4}$, with respective reduced presentations

$$
\begin{gathered}
\left\langle r, s \mid r^{5}=s^{4}=(r s)^{2}=\left(r\left(r s^{-1}\right)^{2}\right)^{2}=1\right\rangle, \\
\left\langle r, s \mid r^{5}=s^{5}=(r s)^{2}=\left(r^{-1} s\right)^{3}=1\right\rangle, \text { and } \\
\left\langle r, s \mid r^{6}=s^{4}=(r s)^{2}=\left(r^{2} s^{2}\right)^{2}=1\right\rangle .
\end{gathered}
$$

For completeness we mention that for $k \geq m$ there are exactly eight noncongruent $(k, m, 2)$-groups of Euler characteristic -2, six of which are automorphism groups of regular maps on orientable surfaces [9].

Having mentioned orientable surfaces, it is appropriate to comment on extendability of methods of this paper to the orientable case, aiming at a classification of regular maps of Euler characteristic $-2 p$ where $p$ is a prime. Unfortunately, in most of our results we use nonorientability in intrinsic ways. An exception is Proposition 3.1 which could be carried over to orientable surfaces by handling the $(k, m, 2)$-groups of Euler characteristic -2 that would arise in extending the proof. Since there has been work in progress by Belolipetsky and Jones [2] towards classification of automorphism groups of Riemann surfaces of genus $p+1$ for prime $p$, we will not pursue this direction here.

\section{Preliminary Reduction Results}

Let $G$ be a $(k, m, 2)$-group of Euler characteristic $-p$ where $p$ is an odd prime. Since $p=-\chi(G)=(1 / 2-1 / k-1 / m)|G| / 2$, it follows that $1 / k+1 / m<1 / 2$; the $(k, m, 2)$-groups satisfying this inequality will be called hyperbolic. Using $\lambda(k, m)=$ $4 k m /(k m-2 k-2 m)$ we see that the order of a hyperbolic $(k, m, 2)$-group $G$ of Euler characteristic $-p$ is given by $|G|=\lambda(k, m) p$.

It is known that for positive integers $k$ and $m$ such that $1 / k+1 / m<1 / 2$ we have $\lambda(k, m) \leq 84$, with equality if and only if $\{k, m\}=\{3,7\}$. The next three largest values of $\lambda(k, m)$ are 48 for $\{k, m\}=\{3,8\}, 40$ for $\{k, m\}=\{4,5\}$, and 36 for $\{k, m\}=\{3,9\}$. We note that the reciprocal of $\lambda(k, m)$ for $1 / k+1 / m<1 / 2$ is proportional to the area of the fundamental region for the action of the extended $(k, m, 2)$-triangle group as a group of isometries in a hyperbolic plane.

Since a computer assisted characterization of all $(k, m, 2)$-groups of (not necessarily prime) Euler characteristic $\chi$ is known [9] for all $\chi \geq-28$, it is sufficient to assume that $p \geq 29$. We use this remark to derive a divisibility condition for the denominator of $\lambda(k, m)$. 
Proposition 3.1. Let $G$ be a hyperbolic $(k, m, 2)$-group of Euler characteristic $-p$ where $p$ is a prime. If $p \geq 29$, then $(k m-2 k-2 m)=p t$ for a positive integer $t$ dividing $4 \mathrm{~km}$, and $|G|=4 \mathrm{~km} / \mathrm{t}$.

Proof. Suppose that $p$ does not divide $k m-2 k-2 m$. Since $|G|=\lambda(k, m) p$ we see that $\lambda(k, m)$ must be an integer, and as $G$ contains the Klein four-group it follows that $4 \mid \lambda(k, m)$. We first claim that $p^{2}$ is not a divisor of $|G|$. Indeed, in the opposite case $p$ would have to divide $\lambda(k, m)$ and so $p \leq \lambda(k, m) / 4 \leq 21$, a contradiction. In particular, since $(k m-2 k-2 m)|G|=4 k m p$ and $p^{2}$ does not divide $|G|, p$ does not divide $k$ or $m$ and the Sylow $p$-subgroups of $G$ are isomorphic to $Z_{p}$.

Suppose now that $H \simeq Z_{p}$ is the unique Sylow $p$-subgroup of $G$. Then $H$ is normal in $G$, and since neither $k$ nor $m$ have $p$ as a factor it follows that the quotient group $G / H$ is a $(k, m, 2)$-group again. From $\lambda(k, m)=|G| / p=|G / H|=$ $-\chi(G / H) \lambda(k, m)$ we see that $\chi(G / H)=-1$. It is known, however, that there are no $(k, m, 2)$-groups of Euler characteristic -1 (see e.g. 9]). Consequently, $G$ contains more than one Sylow $p$-subgroup.

By Sylow theorems, the number of Sylow $p$-subgroups in $G$ is equal to $n p+1$ for some $n \geq 1$ such that $n p+1$ is a divisor of $|G|=\lambda(k, m) p$. This readily shows that $p^{2}<|G|$, so $p<\lambda(k, m)$, which implies that $29 \leq p \leq 83$. But it is easy to check that there are exactly four sets $\{k, m\}$ with $4 \mid \lambda(k, m)$ that satisfy the inequalities $29 \leq p<\lambda(k, m)$, namely, the four sets listed before the statement of Proposition 3.1 .

Out of these sets we can quickly eliminate $\{k, m\}=\{4,5\}$ with $\lambda(4,5)=40$ and $\{k, m\}=\{3,9\}$ with $\lambda(3,9)=36$, since $n p+1$ divides neither $40 p$ nor $36 p$ for any prime $p \geq 29$ and any $n \geq 1$. The only two possibilities for $\{k, m\}=\{3,7\}$ with $\lambda(3,7)=84$ are $p=83$ and $p=41$, giving a potential of two $(2,3,7)$-groups of orders $84 \cdot 83$ and $84 \cdot 41$. However, both can be excluded since by Sylow theorems they contain a unique (and hence normal) cyclic Sylow 7 -subgroup $H$, collapsing in $G / H$ the generator of $G$ of order 7 and thus producing a quotient of too a small order.

It remains to eliminate the case when $\{k, m\}=\{3,8\}$, with $\lambda(3,8)=48$ and $p=47$. We may without loss of generality assume that $G=\langle r, s| r^{3}=s^{8}=$ $\left.(r s)^{2}=\ldots=1\right\rangle$. There must be exactly 48 Sylow 47 -subgroups in $G$, occupying a total of $47^{2}$ elements. This shows that elements of order $2^{i}, 1 \leq i \leq 4$, must be between the remaining 47 elements. Consider the (dihedral) Sylow 2-subgroup $H=\langle s, z\rangle \simeq D_{8}$ of order 16 in $G$. If $H \neq g^{-1} H g$ for some $g \in G$ of order 47 , then all the 47 conjugates of $H$ by powers of $g$ would be pairwise distinct, giving too many elements of order a power of 2 in $G$. It follows that all the $47^{2}$ elements of order 47 normalize $H$, and hence $H$ is normal in $G$. But then the quotient is easily seen to collapse.

It follows that $k m-2 k-2 m=p t$ for a positive integer $t$. From $|G|=\lambda(k, m) p$ we then obtain $|G|=4 \mathrm{~km} / \mathrm{t}$ and so $t$ must be a divisor of $4 \mathrm{~km}$.

For any given prime $p \geq 29$, Proposition 3.1 greatly restricts orders and parameters $k, m$ of the possible $(k, m, 2)$-groups $G$ of Euler characteristic $-p$ : One has to have $|G|=4 \mathrm{~km} / t$ for some $t$ such that $k m-2 k-2 m=t p$. In the next series of three lemmas we prove, besides a number of other facts, that $t \in\{1,2,4\}$. 
Lemma 3.2. Let $G=\langle x, y, z\rangle=\langle r, s\rangle$ be $a(k, m, 2)$-group with $k, m \geq 3$ and let $L=\langle y, z\rangle \cap\langle z, x\rangle$. Then, $L$ is isomorphic to $Z_{2} \times Z_{2}$ if and only if both $k$ and $m$ are even and $r^{k / 2} s^{m / 2} \in\langle z\rangle$; in all other cases $L \simeq Z_{2}$.

Proof. Since $z \in L$ the group $L$ contains a subgroup isomorphic to $Z_{2}$. Assume that $\langle z\rangle$ is a proper subgroup of $L$. Being a subgroup of both $\langle r, z\rangle$ and $\langle s, z\rangle$ the group $L$ must be dihedral. Let $j$ be the smallest positive divisor of $k$ such that $L=\left\langle r^{j}, z\right\rangle$. If $r^{j}$ is not an involution, then it must be in the cyclic subgroup of $\langle s, z\rangle$, that is, $r^{j}=s^{l}$ for some $l$. But from $G=\langle r, s\rangle$ we see that the element $b=r^{j}=s^{l}$ is central in $G$; in particular, $y b y=b$. On the other hand, conjugation by $y$ inverts $b=r^{j}=(y z)^{j}$, and so $b=b^{-1}$, contrary to the fact that $r^{j}$ was assumed to be noninvolutory. Since $k, m \geq 3$, we have $r^{\alpha} \notin\{y, z\}$ for $1 \leq \alpha \leq k-1$ and $s^{\beta} \notin\{x, z\}$ for $1 \leq \beta \leq m-1$. It follows that $k$ must be even and $L=\left\langle r^{k / 2}, z\right\rangle$; by the symmetry of the argument we also have $m$ even and $L=\left\langle s^{m / 2}, z\right\rangle$. This shows that $r^{k / 2} s^{m / 2}$ is either 1 or $z$, and hence is in $\langle z\rangle$. Conversely, if $k$ and $m$ are even and $r^{k / 2} s^{m / 2}$ is in $\langle z\rangle$, then clearly $L$ contains $Z_{2} \times Z_{2}$. That $L=Z_{2} \times Z_{2}$ follows from the beginning of the proof where it is shown that if $r^{j}$ is in $L$, then $r^{j}$ is an involution.

Lemma 3.3. Let $G$ and $L$ be as in Lemma 3.2. If $|G|=k m$, then both $k$ and $m$ are even, $|L|=4$, and $G=\langle y, z\rangle\langle z, x\rangle$. Moreover, $G=\langle r\rangle\langle s\rangle$ if and only if $z=r^{k / 2} s^{m / 2} ;$ otherwise $r^{k / 2}=s^{m / 2}$.

Proof. For any two subgroups $A, B$ of $G$ let $A B$ denote the set of all elements of $G$ that can be written as the product $a b$ where $a \in A$ and $b \in B$; in particular, $A B$ is not necessarily a subgroup of $G$. With the help of the well-known formula $|A B| .|A \cap B|=|A| \cdot|B|$ we obtain $k m=|G| \geq|\langle y, z\rangle\langle z, x\rangle|=|\langle y, z\rangle||\langle z, x\rangle| /|L|=$ $4 \mathrm{~km} /|L|$. Combining with Lemma 3.2 it follows that $|L|=4$ and that both $k, m$ are even. A similar computation shows that $G=\langle r\rangle\langle s\rangle$ if and only if $\langle r\rangle \cap\langle s\rangle=1$. By Lemma 3.2, this is equivalent to $z=r^{k / 2} s^{m / 2}$. The rest is obvious.

Lemma 3.4. Let $G$ and $L$ be as in Lemma 3.2 and suppose that $\chi(G)=-p$ for an odd prime $p$. If $k m-2 k-2 m=t p$, then $t \leq|L|$. In particular, $t \in\{1,2,4\}$. Moreover:

(1) $t=4$ if and only both $k, m$ are even and $(k / 2, m / 2)=1$, and this is also equivalent to $|L|=4$;

(2) $t=2$ if and only if exactly one of $k, m$ is even, with $(k, m)=1$, and then $G=\langle r\rangle\langle z, x\rangle=\langle y, z\rangle\langle s\rangle$;

(3) $t=1$ if and only if both $k$ and $m$ are odd and $(k, m)=1$.

In addition, in the last two cases we have $\langle r\rangle \cap\langle s\rangle=1$.

Proof. We saw earlier that the assumption $k m-2 k-2 m=t p$ implies $|G|=4 k m / t$. By a computation as in the previous proof but with $4 \mathrm{~km} / t$ in place of $\mathrm{km}$ we obtain $4 k m / t=|G| \geq|\langle y, z\rangle\langle z, x\rangle|=|\langle y, z\rangle||\langle z, x\rangle| /|L|=4 k m /|L|$, which shows that $t \leq|L|$. In particular, by Lemma 3.2 we have $t \leq 4$, with $t=4$ implying that $|L|=4$. Conversely, if $|L|=4$, then, invoking Lemma 3.2 again, $k$ and $m$ must be even, say, $k=2 j$ and $m=2 l$. From $t \leq 4$ and $t p=k m-2 k-2 m=4 j l-4 j-4 l$ we see that $t=4$, which gives (1). This also shows that $t<4$ if and only if $|L|=2$, and therefore $t \neq 3$. Parts (2) and (3) follow immediately from the equation $k m-2 k-2 m=t p$ with help of calculations similar to the presented ones. 
Let us emphasize that out of the above three auxiliary results, only the last one depends on tying the parameters $k, m$ with a prime $p$.

For any integer $n$ let $\pi_{n}$, the parity of $n$, be 1 or 2 according to whether $n$ is odd or even. We remark that in terms of parity the three parameters $k, m$ and $t$ from Lemma 3.4 are related by $t=\pi_{k} \pi_{m}$.

We conclude with a few facts that will prove useful in the reduction techniques appearing in the next two sections. First, observe that if $k$ is odd and $(k, m)=1$, then the order of a $(k, m, 2)$-group $G=\langle x, y, z\rangle=\langle r, s\rangle$ is divisible by $4 k m / \pi_{m}$. Indeed, if $m$ is odd, then $|G|$ is divisible by 4 (which is the order of the Klein foursubgroup $\langle x, y\rangle)$ and by the two coprime odd orders $k, m$ of the elements $r, s$. If $m$ is even, then $|G|$ is divisible by the coprime orders $k$ and $2 m$ of the subgroups $\langle r\rangle \simeq Z_{k}$ and $\langle s, x\rangle \simeq D_{m}$.

Lemma 3.5. Let $G=\langle x, y, z\rangle=\langle r, s\rangle$ be a $(k, m, 2)$-group where $k$ is odd and $(k, m)=1$. Let $K$ be a normal subgroup of $G$ such that $K \cap\langle x, y\rangle$ is trivial. Let $\kappa$ and $\mu$ be the orders of the elements $r K$ and $s K$ in $G / K$. Then:

(1) $G / K$ is a $(\kappa, \mu, 2)$-group. Moreover, if $|G|=4 \mathrm{~km} / \pi_{m}$, then $\pi_{\mu}=\pi_{m}$ and $|G / K|=4 \kappa \mu / \pi_{\mu}$ with $K=\left\langle r^{\kappa}\right\rangle\left\langle s^{\mu}\right\rangle$.

(2) In addition, let $K$ be abelian and of odd order. If $m$ is even, then $K$ is a subgroup of $\langle r\rangle$; if $m$ is odd, then $K$ is trivial.

Proof. By the trivial intersection of $K$ with $\langle x, y\rangle$ combined with the above observation, the order of $G / K$ is divisible by $4 \kappa \mu / \pi_{\mu}$. Since the subgroups $\langle r\rangle$ and $\langle s\rangle$ intersect trivially, we have $|K| \geq\left|\left\langle r^{\kappa}\right\rangle\right|\left|\left\langle s^{\mu}\right\rangle\right|=(k / \kappa)(m / \mu)$. From $|G|=4 k m / \pi_{m}$ it then follows that $|G / K|=|G| /|K| \leq 4 \kappa \mu / \pi_{m}$ and hence $\pi_{\mu}=\pi_{m}$ and $|G / K|=$ $4 \kappa \mu / \pi_{\mu}$. This shows that equality must also hold in the above estimates. Therefore $K=\langle u\rangle\langle v\rangle$ where $u=r^{\kappa}$ and $v=s^{\mu}$, and (1) follows.

Now, assume that $K$ is, in addition, abelian and of odd order. Since $\langle u\rangle$ and $\langle v\rangle$ intersect just trivially (Lemma 3.2), for the abelian group $K$ we have $K=\langle u\rangle \times\langle v\rangle$. Normality of $K$ in $G$ together with the fact that $(\kappa, \mu)=1$ imply that $y v y=v^{-j}$ for a suitable $j$. Conjugating this equality by $y$ we obtain $j^{2} \equiv 1(\bmod m / \mu)$. Observe that $r^{-1} v r=z(y v y) z=z v^{-j} z=v^{j}$, and so $r^{-2} v r^{2}=r^{-1} v^{j} r=v^{j^{2}}=v$. It follows that the elements $v$ and $r^{2}$ commute. The fact that $r$ has odd order implies that $v$ commutes with $r$ as well, which shows that $j \equiv 1(\bmod m / \mu)$. Let $y=w(r, s)$, where $w=w(r, s)$ is a word over the alphabet $\{r, s\}$. Note that $w$ commutes with $v$ since both $r$ and $s$ do. But then, $v^{-1}=y v y=w^{-1} v w=v$, which implies $v^{2}=1$. As $|K|$ is odd and $v \in K$, this is possible only if $v=1$ and so $K=\langle u\rangle$. If $m$ is odd, we may interchange the roles of $r$ and $s$ (and of $k$ and $m$ ) in the above arguments to conclude that $K=\langle u\rangle \cap\langle v\rangle$, which is the trivial group. This proves (2).

\section{Hyperbolic $(k, m, 2)$-Groups With Dihedral Sylow two-SubGroups}

Before we consider individual cases for $t \in\{1,2,4\}$ we prove a statement that further substantially restricts potential candidates for our groups. Let $G=\langle x, y, z\rangle=$ $\langle r, s\rangle$ be a hyperbolic $(k, m, 2)$-group, where $k, m \geq 3$ are orders of $r$ and $s$, respectively. Throughout this section we will assume that $k$ is odd.

Let $|G|=4 \mathrm{~km} / \pi_{m}$, where $\pi_{m}$ is the parity of $m$ introduced earlier. Let $c$ be the largest integer such that $2^{c}$ divides $|G|$. If $\pi_{m}=1$, then $c=2$ and $\langle x, y\rangle \simeq Z_{2} \times Z_{2}$ is a Sylow 2-subgroup of $G$. If $\pi_{m}=2$, then $2^{c}$ divides $2 m$, and since $k$ is odd we 
see that $G$ has a dihedral Sylow 2-subgroup contained in $\langle z, x\rangle$. It follows that in any case $G$ has a dihedral Sylow 2-subgroup.

In what follows we will use the following deep result of Gorenstein and Walter (see [16], Part I, Page 85, Theorem 1; for a simplified proof see [3, 4]): If $G$ is a group with a dihedral Sylow 2-subgroup and if $O(G)$ is the (unique) maximal normal subgroup of $G$ of odd order, then $G / O(G)$ is isomorphic to either

(a) a Sylow 2-subgroup of $G$, or

(b) the alternating group $A_{7}$, or

(c) a subgroup of $\operatorname{Aut}(P S L(2, q))$ containing $P S L(2, q), q$ odd.

It is well known that $\operatorname{Aut}(P S L(2, q)), q$ an $n$-th power of an odd prime, is a semidirect product of $P G L(2, q)$ with a cyclic group of order $n$ that comes from the Galois action on the standard matrix representation of $P G L(2, q)$. It follows that any subgroup of $\operatorname{Aut}(\operatorname{PSL}(2, q))$ that contains $P S L(2, q)$ has the form of a split extension $H \rtimes Z_{b}$, where $H$ is either $\operatorname{PSL}(2, q)$ or $P G L(2, q)$ and $b$ is a divisor of $n$. Let $\delta=1 / 2$ or $\delta=1$ according to whether $H \simeq P S L(2, q)$ or $H \simeq P G L(2, q)$, and let $\omega_{1}$ and $\omega_{2}$ be the largest order and the second largest order of an element in $H \rtimes Z_{b}$. Combining the above facts with Dickson's classification [12] of possible orders of elements of $P S L(2, q)$ and $P G L(2, q)$ we conclude the following: If $n \geq 2$, then $\omega_{1}=\delta b(q+1)$ and $\omega_{2}=\delta b(q-1)$, and if $n=1$, then $\omega_{1}, \omega_{2} \in\{\delta(q+1), q\}$.

We are now in a position to prove a structural result about $(k, m, 2)$-groups with dihedral Sylow 2-subgroups.

Proposition 4.1. Let $G=\langle x, y, z\rangle=\langle r, s\rangle$ be a $(k, m, 2)$-group and let $|G|=$ $4 k m / \pi_{m}$, where $k, m \geq 3,(k, m)=1$, and $k$ is odd. Then $G / O(G)$ is isomorphic to $S_{4}$ or $A_{5}$ according to whether $m$ is even or odd.

Proof. As we saw, $G$ has a dihedral Sylow 2-subgroup. By the result of Gorenstein and Walter [16], one of (a), (b), and (c) above holds for $G / O(G)$. We first exclude (a) and (b).

In the case (a) the subgroup $O(G)$ is a normal 2-complement in $G$. It follows that $G$ is a semidirect product of $O(G)$ and a Sylow subgroup $S_{2}$ of $G$. In particular, the element $r$ of odd order $k$ must be in $O(G)$. Let $s=g h$ with $g \in O(G)$ and $h \in S_{2}$ and let $d$ be the order of $h$. Observe that $d<\left|S_{2}\right|$ since the Sylow 2-subgroup is dihedral. But then, $|\langle r, s\rangle| \leq|O(G)| \cdot d<|O(G)|\left|S_{2}\right|=|G|$, a contradiction. The case (b) is easily excluded by observing that the order of $A_{7}$ is much larger than four times the product of orders of any two elements of $A_{7}$. Therefore, for $G / O(G)$ we have the situation described in (c), and it remains to show that $G / O(G)$ is isomorphic to $P G L(2,3) \simeq S_{4}$ or to $P S L(2,5) \simeq A_{5}$ depending on the parity of $m$.

Keeping to the notation introduced before the statement of Proposition 4.1 we have concluded from (c) that $G / O(G) \simeq H \rtimes Z_{b}$, where $H$ is either $P S L(2, q)$ or $P G L(2, q), q$ an $n$-th power of an odd prime, and $b \mid n$. Denoting the cosets of a subgroup of $G$ by bars above the corresponding elements, let $\kappa$ and $\mu$ be the orders of $\bar{r}$ and $\bar{s}$ in $G / O(G)=\langle\bar{r}\rangle\langle\bar{z}, \bar{x}\rangle$; clearly $\kappa \neq \mu$. Using part (1) of Lemma 3.5, for $n \geq 2$ we obtain $\delta q\left(q^{2}-1\right) b=\left|H \rtimes Z_{b}\right|=|G / O(G)|=4 \kappa \mu / \pi_{\mu} \leq 4 \delta b(q+1) \delta b(q-1)$. This implies that $q \leq 4 \delta b \leq 4 n$, which is absurd for any $n$-th power $q$ of any odd prime if $n \geq 2$. For $n=1$ we obtain $\delta q\left(q^{2}-1\right)=|H|=|G / O(G)|=4 \kappa \mu / \pi_{\mu} \leq$ $4 \delta q(q+1) / \pi_{m}$. If $m$ is even, this gives a contradiction for all odd $q \geq 5$ but is feasible if $q=3$ (for both values of $\delta$ ); if $m$ is odd, then we have a contradiction for all odd $q \geq 7$ but feasibility for $q=3$ or $q=5$. 
For even $m$ it follows that $G / O(G)$ is isomorphic to either $P S L(2,3) \simeq A_{4}$ or $P G L(2,3) \simeq S_{4}$. The group $A_{4}$, however, cannot be generated by three involutions and hence $G / O(G) \simeq S_{4}$, as claimed. For odd $m$ and $q=3$ we have only the groups $P S L(2,3) \simeq A_{4}$ and $P G L(2,3) \simeq S_{4}$ as candidates for $G / O(G)$, neither of which can be generated by two elements of coprime odd orders. Finally, if $m$ is odd and $q=5$, then $G / O(G)$ can only be isomorphic to $P S L(2,5) \simeq A_{5}$ or to $P G L(2,5) \simeq S_{5}$. But the only two nontrivial coprime odd orders here are 3 and 5 and the corresponding permutations in $S_{5}$ are even, showing that $G / O(G) \simeq A_{5}$ in this case.

\section{Further Reduction Results}

We begin by analyzing the individual cases $t=1,2$, and 4 . Let us first point out that the three values of $t$ came out as a consequence of the parameters $k$ and $m$ being tied with a prime number $p$ by the equation $k m-2 k-2 m=t p$. The three reduction results below, however, are valid for any $k$ and $m$ as stated, with no reference to any prime $p$.

If $t=1$, then, by part (3) of Lemma 3.4, we have $|G|=4 \mathrm{~km}$ with both $k$ and $m$ odd, and $(k, m)=1$. In this situation, Proposition 4.1 can be strengthened as follows.

Proposition 5.1. Let $G=\langle x, y, z\rangle=\langle r, s\rangle$ be a $(k, m, 2)$-group with $|G|=4 k m$, where $k, m \geq 3$, both $k$ and $m$ are odd, and $(k, m)=1$. Then $G \simeq A_{5}$.

Proof. Let $G$ be a counterexample of the smallest order. From Proposition 4.1 it follows that $G / O(G) \simeq A_{5}$, and so $|O(G)| \geq 3$. We first show that no nontrivial proper subgroup of $O(G)$ is normal in $G$. Indeed, if $K$ was such a subgroup, then, by part (1) of Lemma 3.5 the group $G / K$ would satisfy the assumptions of the theorem, and from $60<|G / K|<|G|$ we would obtain a contradiction with minimality of $|G|$.

Without loss of generality we may suppose that $G / O(G)=\langle\bar{r}, \bar{s}| \bar{r}^{5}=\bar{s}^{3}=$ $\left.(\bar{r} \bar{s})^{2}=1\right\rangle \simeq A_{5}$. Invoking part (1) of Lemma 3.5 again, we have $O(G)=\left\langle r^{5}\right\rangle\left\langle s^{3}\right\rangle$, a product of two cyclic groups. By Ito's theorem [19], the commutator subgroup $K=[O(G), O(G)]$ is a proper characteristic subgroup of $O(G)$, and so $K$ is normal in $G$. The observation in the preceding paragraph then implies that $K=1$, that is, $O(G)$ is abelian. Since both $k$ and $m$ are odd, part (2) of Lemma 3.5 applied to the subgroup $O(G)$ shows that $O(G)$ is trivial. This contradiction finishes the proof.

Let us now consider the situation when $t=2$. By part (2) of Lemma 3.4 we know that $G=\langle r\rangle\langle z, x\rangle=\langle y, z\rangle\langle s\rangle$. From the same source it follows that exactly one of $k$ and $m$ is even, and $(k, m)=1$. Without loss of generality we may assume that $m$ is even.

Proposition 5.2. Let $G=\langle x, y, z\rangle=\langle r, s\rangle$ be a hyperbolic $(k, m, 2)$-group such that $|G|=2 k m$ where $k$ is odd, $m$ is even, and $(k, m)=1$. Then, $k=3 j$ with $j$ odd, $m=4$, and $G=\langle r\rangle\langle z, x\rangle \simeq Z_{k} \cdot D_{4}$, with reduced presentation

$$
\left\langle r, s \mid r^{k}=s^{4}=(r s)^{2}=s r^{3} s^{-1} r^{3}=1\right\rangle .
$$

Proof. According to Proposition 4.1 we know that, without loss of generality, $G / O(G)=\left\langle\bar{r}, \bar{s} \mid \bar{r}^{3}=\bar{s}^{4}=(\bar{r} \bar{s})^{2}=1\right\rangle \simeq S_{4}$. Also, part (1) of Lemma 3.5 shows 
that $O(G)=\left\langle r^{3}\right\rangle\left\langle s^{4}\right\rangle$. By induction on $|G|$ we prove that $s^{4}=1$, that is, $m=4$. Indeed, let $G$ be a group as above such that 24 properly divides $|G|$. If $O(G)$ is abelian, then from $s^{4} \in O(G)$ and from part (2) of Lemma 3.5] we deduce that $s^{4}=1$. In the case when $O(G)$ s not abelian, then (by Ito's theorem) the commutator subgroup $K$ of $O(G)$ is abelian. By induction, in $G / K$ we have $(s K)^{4}=1$, that is, $s^{4} \in K$. Being characteristic in $O(G)$ the subgroup $K$ is normal in $G$. Invoking part (2) of Lemma 3.5 again and applying it to the abelian normal subgroup $K$ of odd order we conclude that $s^{4}=1$. It follows that $m=4$ as claimed, and therefore $O(G)=\left\langle r^{3}\right\rangle$. Moreover, since $\langle z, x\rangle \simeq D_{4}$, from $|G|=2 k m=8 k$ we see that $G=\langle r\rangle\langle z, x\rangle \simeq Z_{k} \cdot D_{4}$.

It remains to address the presentation of $G$. From $\left\langle r^{3}\right\rangle=O(G)$ we see that $k=3 j$ for some odd $j$. By normality of $O(G)$ in $G$ we have $x r^{3} x=r^{3 i}$ (and hence $s r^{3} s^{-1}=r^{-3 i}$ as well) for some $i$ such that $i^{2} \equiv 1(\bmod j)$. Let $x=w(r, s)$, where $w=w(r, s)$ is a word in $r$ and $s$. If $s$ occurs in $w$ an odd number of times, then $r^{3 i}=x r^{3} x=w r^{3} w=r^{-3 i}$ since $i^{2} \equiv 1(\bmod j)$, which quickly contradicts the fact that $r$ has odd order. Therefore, $s$ occurs in $w$ an even number of times, and by the same token we have $r^{3 i}=x r^{3} x=w r^{3} w=r^{3}$, implying that $i=1$.

Finally, we are ready to deal with the case $t=4$. Part (1) of Lemma 3.4 tells us that we only need to consider $k$ and $m$ such that $(k, m) \leq 2$.

Proposition 5.3. Let $G=\langle x, y, z\rangle=\langle r, s\rangle$ be a hyperbolic $(k, m, 2)$-group with $|G|=k m$, where $(k, m) \leq 2$. Then $k$ and $m$ are even, $k / 2$ and $m / 2$ are odd, and $G=\left\langle y, r^{2}\right\rangle \times\left\langle x, s^{2}\right\rangle \simeq D_{k / 2} \times D_{m / 2}$ has reduced presentation

$$
\left\langle r, s \mid r^{k}=s^{m}=(r s)^{2}=\left(r s^{-1}\right)^{2}=1\right\rangle .
$$

Proof. Invoking Lemma 3.3 we see that $|G|=k m$ implies $|L|=4$, with $G=$ $\langle y, z\rangle\langle z, x\rangle$. First, assume that $G=\langle r\rangle\langle s\rangle$, which (by Lemma 3.3 again) implies $k=2 j, m=2 l$, and $z=r^{j} s^{l}$.

By Ito's theorem [19], the commutator subgroup $G^{\prime}=[G, G]$ of $G$ is abelian. Note that both $r^{2}=[y, z]$ and $s^{2}=[z, x]$ are in $G^{\prime}$. Clearly $(j, l)=1$; we show that both $j$ and $l$ are odd. Indeed, if, say, $j$ is even, then $l$ must be odd. Right multiplication of both sides of $z=r^{j} s^{l}$ by $s$ then gives $x=r^{j} s^{l+1} \in G^{\prime}$. In particular, as both $x$ and $s^{2}$ belong to the abelian $G^{\prime}$, we have $x s^{2} x=s^{2}$. But conjugation by $x$ obviously inverts $s^{2}$, and so $s^{2}=s^{-2}$ and hence $l \leq 2$, a contradiction.

Having both $j, l$ odd we see that $r z s=r^{j+1} s^{l+1} \in G^{\prime}$. Since $r z s=y z x=y s=$ $r x$ is in the abelian subgroup $G^{\prime}$, we have $(y s) s^{2}=s^{2}(y s)$ and $(r x) r^{2}=r^{2}(r x)$. It follows that $y$ commutes with $s^{2}$ and $x$ with $r^{2}$. Since conjugation by $y$ and $x$ inverts $r^{2}$ and $s^{2}$, respectively, we see that $G$ is a direct product of the dihedral subgroups $\left\langle y, r^{2}\right\rangle \simeq D_{j}$ and $\left\langle x, s^{2}\right\rangle \simeq D_{l}$. The last relator in the presentation of $G$ is equivalent with $y$ and $x$ commuting with $s^{2}$ and $r^{2}$, respectively, and the completeness of the presentation is easy to check.

To finish the proof we have to analyze the situation when $|G|=k m$ and $G \neq$ $\langle r\rangle\langle s\rangle$. From Lemma 3.2 and Lemma 3.3 we see that in this case $k=2 j, m=2 l$, and $b=r^{j}=s^{l}$ is a central involution of $G$. Then, the quotient group $G /\langle b\rangle$ of order $k m / 2=2 j l$ where $(j, l)=1$, has presentation $\bar{r}^{j}=\bar{s}^{l}=(\bar{r} \bar{s})^{2}=\ldots=1$. Observe that $j, l \geq 3$; otherwise one of $j, l$ would be equal to 2 and then the three relations in the preceding presentation would define a dihedral group of order $j l$, a 
contradiction. Since $(k, m) \leq 2$, both $j$ and $l$ cannot be even; say, $j$ is odd. It can be checked that $b \notin\langle x, y\rangle$. Following part (1) of Lemma 3.5, $G /\langle b\rangle$ is a $(j, l, 2)$-group, and so its order $2 j l$ is divisible by 4 , showing that $l$ is even. From Proposition 5.2 we then see that $l=4, j=3 i$ for some odd $i$, and

$$
G /\langle b\rangle=\left\langle\bar{r}, \bar{s} \mid \bar{r}^{3 i}=\bar{s}^{4}=(\bar{r} \bar{s})^{2}=\bar{s} \bar{r}^{3} s^{-1} \bar{r}^{3}=1\right\rangle \simeq Z_{j} \cdot D_{4} .
$$

Moreover, centrality of $b$ in $G$ and presentation of $G /\langle b\rangle$ allows us to conclude that the original group $G$ must have had a full presentation of the form $r^{6 i}=s^{8}=$ $(r s)^{2}=r^{3 i} s^{4}=x r^{3} x r^{-3} r^{3 \alpha}=1$ where $\alpha=0$ or $\alpha=i$. This shows that, in any case, $\left\langle r^{6}\right\rangle$ is a normal subgroup of $G$ of (odd) order $i$. The corresponding quotient group $G /\left\langle r^{6}\right\rangle$ of order $|G| / i=48$ then must be a $(6,8,2)$-group of Euler characteristic $\chi\left(G /\left\langle r^{6}\right\rangle\right)=(1 / 6+1 / 8-1 / 2) \cdot 24=-5$. But by the list of Conder and Dobcsányi [9], no such group exists.

\section{Proof of The MAIN RESUlt AND REMARKS}

With the help of results established in the previous section we are in a position to prove our main result.

Proof of Theorem 2.2. Let $G=\langle x, y, z\rangle=\langle r, s\rangle$ be a $(k, m, 2)$-group of characteristic $p$, where $p$ is an odd prime and $k \geq m$. We know that such a group must be hyperbolic. The part of the list of groups in the statement of Theorem 2.2 corresponding to $p \leq 23$ is extracted from the classification of $(k, m, 2)$-groups of Euler characteristic at least -28 , given in 9]. We may therefore assume that $p \geq 29$. By Proposition 3.1 and Lemma 3.4 we have $k m-2 k-2 m=t p$ and $|G|=4 k m / t$, where $t \in\{1,2,4\}$.

If $t=1$, then we have $k m-2 k-2 m=p$ and hence both $k$ and $m$ are odd and $(k, m)=1$. By Proposition 5.1 up to isomorphism there is a unique such $(k, m, 2)$ group of order $4 \mathrm{~km}$, namely, the $(5,3,2)$-group isomorphic to $A_{5}$. But this group has Euler characteristic 1, a contradiction.

Now let $t=2$. From $k m-2 k-2 m=2 p$ it follows that exactly one of $k, m$ is odd and $(k, m)=1$. Proposition 5.2 then shows that (up to isomorphism) we have a unique $(k, m, 2)$-group $G$ of order $2 k m$ here, with $k=3 j$ for an odd $j$, and $m=4$. Invoking the equation $k m-2 k-2 m=2 p$ again we see that this is possible only if $p \equiv-1(\bmod 3)$ and $k=p+4$. Therefore, $G$ is the $(p+4,4,2)$-group $G_{p}$ that appears in parts (3) and (6) of Theorem 2.2.

In the last case when $t=4$, from $k m-2 k-2 m=4 p$ we obtain $(k, m) \leq 2$. According to Proposition [5.3 we have $k=2 j$ and $m=2 l$ where $j>l$, both $j$ and $l$ are odd, and $(j, l)=1$. Then, $k m-2 k-2 m=4 p$ is equivalent with $(j-1)(l-1)=p+1$ and hence $p \equiv-1(\bmod 4)$. Further, by Proposition 5.3, for each such pair $(j, l)$ there is (up to isomorphism) a unique $(2 j, 2 l, 2)$-group of order $\mathrm{km}=4 j l$, namely, the group $G_{j, l}$. Recalling the function $\nu$ introduced in Section 2 we have a total of $\nu(p)$ noncongruent groups in this case, featuring in parts (4) and (6) of Theorem 2.2 .

Thus, for $p \geq 29,(k, m, 2)$-groups of a negative prime Euler characteristic $-p$ exist if and only if $p \equiv-1(\bmod 3)$ or $p \equiv-1(\bmod 4)$. It follows that for primes $p \geq 29$ there are no $(k, m, 2)$-groups of Euler characteristic $-p$ if $p \equiv 1(\bmod 12)$, which is part (1) of Theorem 2.2 Parts (3), (4) and (6) of Theorem 2.2 for $p \geq 29$ are immediate consequences of the foregoing analysis. 
Uniqueness of the $(k, m, 2)$-groups whose reduced presentations are given as in Theorem 2.2 follows from our proof in Sections 4 and 5. An independent argument can be given by observing that the centers of our groups are trivial and quoting Theorem 7.7 from [28] that can be restated as follows: If a group

$$
G=\left\langle r, s \mid r^{k}=s^{m}=(r s)^{2}=\ldots=1\right\rangle
$$

has center of an odd order, then $G$ has (up to congruence) a unique representation as a $(k, m, 2)$-group.

We conclude with a few remarks regarding the map-theoretic interpretation of our main result. Since the automorphism group of a regular map acts transitively on flags and also on directed edges, regular maps always have simple quotient maps, such as a single semi-edge in a sphere or in a disc, depending on whether the map is orientable or not. In general, regular quotient maps are obtained precisely by dividing out by normal subgroups of the automorphism group; the smaller the subgroups, the larger the quotients.

From the presentation of the group $G_{p}$ for prime $p \equiv-1(\bmod 3)$ it is clear that $G_{p}$ contains a cyclic normal subgroup of order $(p+4) / 3$ generated by $r^{3}$. The quotient $G_{p} /\left\langle r^{3}\right\rangle \simeq\left\langle\bar{r}, \bar{s} \mid \bar{r}^{3}=\bar{s}^{4}=(\bar{r} \bar{s})^{2}=1\right\rangle$ is the automorphism group of a regular embedding $M$ of a complete graph of order four in a projective plane. It follows that the regular maps corresponding to the groups $G_{p}$ are cyclic normal $(p+4) / 3$-fold covers of $M$, with branch points of order $(p+4) / 3$ at each of the four vertices of $M$. An analogous statement can be made about the dual maps.

Similarly, for primes $p \equiv-1(\bmod 4)$ the groups $G_{j, l}$ associated with $p$ contain a normal subgroup isomorphic to $Z_{j} \times Z_{l}$ generated by $r^{2}$ and $s^{2}$. The reduced presentation $\left\langle\bar{r}, \bar{s} \mid \bar{r}^{2}=\bar{s}^{2}=(\bar{r} \bar{s})^{2}=1\right\rangle \simeq Z_{2} \times Z_{2}$ of the resulting quotient group represents a regular projective-planar embedding $M^{\prime}$ of a loop with a single vertex. We conclude that the $\nu(p)$ regular maps corresponding to the groups $G_{j, l}$ are normal $Z_{j} \times Z_{l}$-covers of $M^{\prime}$, with the two branch points being the vertex (of order $j$ ) and the center of the single face (of order $l$ ). More statements of this type can be generated by considering other normal subgroups of $G_{j, l}$.

Regular maps corresponding to the groups of Theorem 2.2 can be lifted from nonorientable supporting surfaces $\mathcal{N}_{p+2}$ to their orientable double covers. Our reduced presentations thus automatically give a classification of antipodal regular maps on orientable surfaces of genus $p+1$ for prime $p$. This may be beneficial for possible future projects regarding classification of regular maps on orientable surfaces. More details on antipodality can be found in [28].

Finally, we note that by [33] there are no regular maps on nonorientable surfaces of Euler characteristic $-16,-22,-25$ and -46 , which are values not covered by part (1) of Theorem 2.2. The problem of determining all nonorientable surfaces that do not support any regular map remains open.

\section{REFERENCES}

1. R. D. M. Accola, On the number of automorphisms of a closed Riemann surfaces, Trans. Amer. Math. Soc. 131 (1968), 398-408. MR36:5333

2. M. Belolipetsky and G. Jones, Automorphism groups of Riemann surfaces of genus $p+1$, where $p$ is a prime, submitted.

3. H. Bender, Finite groups with dihedral Sylow 2-subgroups, J. Algebra 70 (1981), 216-228. MR83c:20011b 
4. H. Bender and G. Glauberman, Characters of finite groups with dihedral Sylow 2-subgroups, J. Algebra 70 (1981), 200-215. MR83c:20011a

5. P. Bergau and D. Garbe, Non-orientable and orientable regular maps, in: Proceedings of "Groups-Korea 1988", Lect. Notes Math. 1398, Springer (1989), 29-42. MR90k:57003

6. H. R. Brahana, Regular maps and their groups, Amer. J. Math. 49 (1927), 268-284.

7. R. P. Bryant, D. Singerman, Foundations of the theory of maps on surfaces with boundary, Quart. J. Math. Oxford Ser. (2) 36 (1985), no. 141, 17-41. MR86f:57008

8. W. Burnside, "Theory of Groups of Finite Order", Cambridge Univ. Press, 1911.

9. M. Conder and P. Dobcsányi, Determination of all regular maps of small genus, J. Combinat. Theory Ser. B 81 (2001), 224-242. MR2002f:05088

10. M. Conder and B. Everitt, Regular maps on non-orientable surfaces, Geom. Dedicata 56 (1995), 209-219. MR96g:05046

11. H. S. M. Coxeter and W. O. J. Moser, "Generators and Relations for Discrete Groups", 4th Ed., Springer-Verlag, Berlin, 1984. MR81a:20001

12. E. Dickson, "Linear groups with an exposition of Galois field theory", 1901; Dover Publ., 1958. MR21:3488

13. W. Dyck, Über Aufstellung und Untersuchung von Gruppe und Irrationalität regularer Riemannscher Flächen, Math. Ann. 17 (1880), 473-508.

14. D. Garbe, Über die regulären Zerlegungen geschlossener orientierbarer Flächen, J. Reine Angew. Math. 237 (1969), 39-55. MR39:7502

15. A. Gardiner, R. Nedela, J. Širán̆ and M. Śkoviera, Characterization of graphs which underlie regular maps on closed surfaces, J. London Math. Soc. (2) 59 (1999) No. 1, 100-108. MR2000a:05104

16. D. Gorenstein and J. H. Walter, The characterization of finite groups with dihedral Sylow 2-subgroups, I, II, III, J. of Algebra 2 (1965), 85-151, 218-270, 334-393. MR31:1297a. MR 31:1297b MR 32:7634

17. A. Grothendieck, "Esquisse d'un programme", Geometric Galois actions, London Math. Soc. Lecture Note Ser., No. 242, Cambridge Univ. Press, Cambridge, 1997, pp. 1, 5-48. MR99c:14034

18. L. Heffter, Uber metazyklische Gruppen und Nachbarconfigurationen, Math. Ann. 50 (1898), 261-268.

19. N. Ito, Über das Produkt von zwei abelschen Gruppen, Math. Z. 62 (1955), 400-401. MR 17:125b

20. L. D. James and G. A. Jones, Regular orientable imbeddings of complete graphs, J. Combinat. Theory Ser. B 39 (1985), 353-367. MR87a:05060

21. G. A. Jones, Maps on surfaces and Galois groups, Math. Slovaca 47 (1997), 1-33. MR98i:05055

22. G. A. Jones and D. Singerman, Theory of maps on orientable surfaces, Proc. London Math. Soc. (3) 37 (1978), 273-307. MF 58:21744

23. G. A. Jones and D. Singerman, Belyı functions, hypermaps, and Galois groups, Bull. London Math. Soc. 28 (1996), 561-590. MR97g:11067

24. J. Kepler, "The harmony of the world" (translation from the Latin "Harmonice Mundi", 1619), Memoirs Amer. Philos. Soc. 209, American Philosophical Society, Philadelphia, PA, 1997. MR2000c:01020

25. F. Klein, Uber die Transformation siebenter Ordnung der elliptischen Functionen, Math. Ann. 14 (1879), 428-471.

26. C. MacLachlan, A bound for the number of automorphisms of a compact Riemann surface, J. London Math. Soc. 44 (1969), 265-272. MR38:4674

27. C. L. May and J. Zimmerman, There is a group of every strong symmetric genus, Bull. London Math. Soc. 35 (2003), 433-439. MR2004b:57026

28. R. Nedela and M. Škoviera, Exponents of orientable maps, Proc. London Math. Soc. (3) 75 (1997), 1-31. MR 98i:05059

29. C. H. Sah, Groups related to compact Riemann surfaces, Acta Math. 123 (1969), 13-42. MR 40:4447

30. F. A. Sherk, The regular maps on a surface of genus three, Canad. J. Math. 11 (1959), 452-480. MR21:5928

31. T. W. Tucker, Finite groups acting on surfaces and the genus of a group, J. Combinat. Theory Ser. B 34 (1983) No. 1, 82-98. MR85b:20055 
32. W. T. Tutte, What is a map?, in "New Directions in Graph Theory" (F. Harary, Ed.), Acad. Press, 1973, 309-325. MR 51:12589

33. S. Wilson and A. Breda D'Azevedo, Surfaces with no regular hypermaps, Discrete Math. 277 (2004), 241-274.

Departamento de Matematica, Universidade de Aveiro, Aveiro, Portugal

E-mail address: breda@mat.ua.pt

Institute of Mathematics, Slovak Academy of Science, Banská Bystrica, Slovakia

E-mail address: nedela@savbb.sk

Department of Mathematics, Svf, Slovak Univ. of Technology, Bratislava, Slovakia

E-mail address: siran@math.sk 